\title{
Wiedemann-Steiner syndrome
}

INSERM

\section{Source}

INSERM. (1999). Orphanet: an online rare disease and orphan drug data base.

Wiedemann-Steiner syndrome. ORPHA:319182

Wiedemann-Steiner syndrome is a rare, genetic multiple congenital

anomalies/dysmorphic syndrome characterized by short stature, hypertrichosis cubiti,

facial dysmorphism (hypertelorism, long eyelashes, thick eyebrows, downslanted,

vertically narrow, long palpebral fissures, wide nasal bridge, broad nasal tip, long

philtrum), developmental delay, and mild to moderate intellectual disability. It has a

variable clinical phenotype with additional manifestations reported including muscular

hypotonia, patent ductus arteriosus, small hands and feet, hypertrichosis on the back, behavioral difficulties, and seizures. 